\title{
Klaus Beer Sozial-liberale Justizreform - eine Bilanz
}

Ich sichre die Ergebnisse der sozialliberalen Justizreform der siebziger Jahre und ziche Bilanz. Nach einem Blick auf dic Ausgangslage werden die konkreten Reformschritce beschrieben. Sodann wird der erreichte Stand am Ende der Reformepoche geschildere und kommentiert und den anfänglichen Absichocn und Errvartungen gegenübergestellt. Aus der Berufstäcigkeit des Betracheers als Zivilrichter crgibe sich, daß bei dem Bericht über die Reformen der Zivilprozeß in den Vordergrund tritt. Der Leser möge beuricilen, wieweir die Richtigkeit der allgemeinen Schlußfolgerungen dadurch berührt wird.

Wozu und für wen cine solche Bilanz? In erster Linic entspringt sie dem Bedürfnis nach Diskussion in Kreisen kricischer Juristen über die gegen wärtige Lage der Justiz. Die jüngeren Kollegen haben die Reformphase ganz oder zum Tcil noch nicht als berufstätige Juristen erlebt. Es bilden sich schncll Legenden über die Vergangenheit. Geschönte und gehässige Schlußstriche unter dic Reformen werden gezogen. Absichcen für die Zukunf́ werden motiviert, erklärt und propagicrt mit wirklichen oder angeblichen Erfahrungen in den siebziger Jahren. Sichten und prüfen wir also selbsi.

Interessant sind auch Vergleiche der Entwicklungen in der Bundesrepublik mit Abläufen im Ausland. Etwa mit Frankreich, an dessen ganz andersartiger "Wende die französischen Richtcrkollegen im Syndicat de la Magistrature hochfliegende Erwartungen knüpiren. Oder mir Iralien, wo die Richrer der Magistratura Democratica die Bilanz aus dem whistorischen Kompromiß wcrden ziehen müsscn. Diskutieren wir mit ihnen, muß uns klar sein, was bei uns die Reformen gebracht haben.

\section{Die Ausgangslage}

Neben Problemen der Arbeitsbeschaffung und Wirtschafisplanung wurden Mitic und Ende der sechziger Jahre Themen der Bildung und der Rechtspolitik zentrale Inhalt von Reformpolitik. Die Impulse zur Justizkririk und -polirik gingen stark in die Policik der SPD ein. Bedeutender Vertreter unter den Reformern war Rudolf Wassermann, wichriges Diskussionsforum die Arbeitsgemeinschaft sozialdemokratischer Juristen (ASJ). Dokumente der damaligen Reformforderungen sind beispielsweise die Leitsärze dieser Arbcitsgemeinschaft zur Juscizpolitik' und das nKönigsteiner Manifest * des Akrionskomitees Justizreform². Viele anderc damalige Äuße-

I Rech( und Policik) 968, S. 107

2 In: R. Whsemann (Hrso), Juscizrefom, Neuwied 1968, S. 178. 
rungen zur Justizreform sind an diese Aktivitäten schr stark angelehnt, z. B. diejenigen der politischen Parteien und des Deurschen Richterbundes.

Die ASJ-Leitsärze forderzen, daß die Jusciz dem Bürger hilft, seine Grundrechte durchzusetzen; die Justiz soltte die Staatsgewalt begrenzen, dic Chancengleichheit benachieiligter Bürger herstellen und helfen, soziale Gcrechrigkeir herbeizuführen. Um die Justiz dazu zu befähigen, wurde eine Reform der Juriscenausbildung vorgeschlagen, ergänzr durch eine Reform der Justiz. In diesem Zusammenhang war im einzelnen vorgesehen:

- Auflösung der überkommenen bcamtenrechclichen Hierarchie in der Weise, daß die Richter der beiden ersten Instanzen gleiche Titel tragen und gleiche Besoldung crhalten;

- Wechsel im Vorsizz der Richcerkollegien, Zulassung abwcichender Meinungen im Urccil;

- Beteiligung der Richter an der Justiz- und Gerichtsverwaltung und Venretungsmöglichkcit von Etatforderungen ohne Zwischenschaltung des Justizministeriums gegenüber dem Parlament (instizutionalisiert in Wege von Parlamentsausschüssen);

- Auflösung der Vierstufigkeit der Jusciz (Amrs-, Land-, Oberlandesgericht und Bundesgerichishof) zugunsten von drei Instanzen, die das Gerichtswesen transparenter und zugänglicher machen sollte.

Rudolf Wassermann wurde nicht müde, für einen kompensatorischen Verhandlungsstil einzucreten, der dic Chancengleichheir vor Gericht gewährleister. Er machte deurlich, $d_{a} ß$ Richter politisch handeln, wenn sie sich den sozialstaatlichen Herausforderungen des Grundgesetzes sicllen und öffnen, aber auch, wenn sie - in überkommenen Vorurteilen befangen - das verweigern. Er forderte die Richter auf, den politischen Charakter ihres Berufes zu crkennen und sich den Reformforderungen nichr zu verschließen. Als Ziel der Justizreformen schwebren ihm wmöglichst wenig Inhumanität, Diskriminierung und Selbstentfremdung und moglichst viel konkrete Humanität, realc Selbstbestimmung und sozialc Gerechtigkeir" vor.'

Schon darmals gab es aber auch Varianten der Justizkricik und Änderungsvorschläge, die nicht praktisch wurden, weil sie in eine etwas andere Richtung wiesen. Theo Rasehorn beschrieb die auf Hilfe angewicsenen Unterschichten weniger abstrakt: ungelernte Arbeiter, Subproletariat, sozial Deklassierre, Ausländer, die Bevölkerung von Obdachlosen-Ghetros, Kriminelle und Vorbestrafte, gefälrdere Jugendliche, Alte, Behinderte. Entsprechend konkrer stellte er sich die Beratung, Hilfe, Vermittlung und Betreuung vor, welche dic Justiz ihnen zukommen lassen sollee: in engem Kontakt, in klcinen Sprengeln sollten die Gerichte ihnen entgegenkommen und offenscehen, unbürokratisch und unspezialisiert. Über diese Vorstellungen ${ }^{4}$ ging die zur Tar drängende Justizreform hinweg. Zur Vorbereitung der Dreistufigkeir (also der Zusammenlegung der Amrs- und Landgerichte) wurden allenthalben die klcinen Amtsgerichce aufgehoben und die Gerichrssprengel vergrößert. Das Richterpersonal an den verbleibenden Amesgerichten war dadurch zahlreicher und spezialisierter. Das sollte auch der Effekrivität und Qualitär der richterlichen Arbeit dicnen.

Rasehorns Ansichten waren insgesamt skeprischer, seinc Ziele warcn weniger ausgreifend und bescheidener als das, was ab ig 99 zur Regierungspolitik wurde. Wieder andere Justizkritiker führcen die starke Bewegung in den Köpín kritischer Justiz-

3 Richter, Reform, Gesellschaft, Ncuwied ig70, S. 22.

4 Th. Raschorn: Kritische Jusciz $1969,5273,28_{1}$; Bcierag ${ }_{4}$ Opas Justiz. Icber in: U. Sonncmann (Hrsg.), Wie frei ist unsere Justix? München r969, S. 13, 25; Rexht und Klassen. Nouwied 1974, S. 102 ff. 
juristen nur auf den Legitimationszwang der Klassenjustiz in Zciten des offiziellen Versagens der Politik vor den Ansprüchen der Zcit zurück.

Eine Schwäche der vorherrschenden Reformvorstellungen waren jedenfalls von vornherein ihre blassen, unkonkreten, pauschalen gesellschafclichen Ziclseczungen. Es blieb bei großen, allgemeinen und plakativen Gemeinwohlvorstellungen zugunsten des breiten Volkes, ohne daß erkennbar war, welche konkreten gescllschafclichen Entwicklungen die Justizreformen unterstützen konnten. Viele fortschritshungrige Justizkritiker erkannten sich aber dennoch in den Reformbestrebungen wieder, die viele Illusionen hinsichtlich ihrer Möglichkeiten und ihrer policischen Tragweite weckten. Darin scheint von Anfang an die Gefahr von Encräuschungen gelegen zu haben, wenn die Reformen schließlich nicht verwirklicht wurden.

\section{Realisierte Reformen}

Zur Juscizreform) gehören zunächst diejenigen Änderungen, wclche den Justizapparat selbst und sein Verhältnis zum Publikun zum Gegenstand haben. Aber auch die geserzliche Regelung gesellschaftlicher Problembereiche außerbalb der Justiz kann die Justiz ändern, wenn dic Einwirkung auf die Gesellschafr in hohem Maße justizvermitrelr erfolgr: wenn der Vollzug oder die Sicherung der Reform im wesentlichen durch Justiztätigkeit geschieht und auch auf die Justiz wirkt.

Zur Justizreform im ersten, direkten Sinn kann man die Verbesserung der Mitwirkungsrechte der Richter innerhalb der Justiz, die Verbesserung der Richterbesoldung, die Änderung der Richtertitel, die Umstrukturierung der Juristenausbildung, die Abschaffung der kleinen Amesgerichre, die Gerichrsscandsnovelle, die Enclaszungsnovelle, die Kostenrechtsnovelle, die Vereinfachungs- und Bcschleunigungsnovelle, das Beratungshilfegesetz und das Prozeßkostenliilfegesetz rechnen.

$\mathrm{Zu}$ den justizvermittelten Rechtsreformen gehören die Änderung des Verbraucherrechis, hauptsächlich des Abzahlungsgeserzes, ferner das Gesetz über Allgemeine Geschäfrsbedingungen, die Familienrechtsreformen und die Strafrechtsreformgesetze. Um am Ende der Reformepoche meine Justiz-Bilanz plausibel zu machen, rufe ich dic wichrigsten Schritte der Justizreform im direkten, engeren Sinne ins Gedächtnis.

1. Die Beteiligung der Richter an der Entscheidung ihrer Angelegenheiten durch die Justizverwaltung ist in verschiedene Organe aufgespalten: Die Vertcilung der Richter und ihrer Arbeit auf die Spruchkörper (oder Einzelrichrer-Dezernate) besorgen die Gerichsspräsidien; dic Berciligung an den Ernennungen und Bcförderungen von Rıchtern licgr bei den Präsidiairäten der verschiedenen Gerichrszweige; die Beveiligung der Richter an allen übrigen allgemeinen und sozialen Angelegcnheiten nehmen die den Personalräten ähnelnden Richterräre rvahr.

Im Nationalsozialismus war die Geschäftsverteilung seit 1937 Sache der Gerichrspräsidenten. Das Vereinheitlichungsgesetz vom 12.9 . 1950 stellte die Zuständigkeit der Präsidien der ordentlichen Gerichte wieder her. Das Präsidium z. B. eines Landgeriches bestand hinfort aus dem Präsidenten als Vorsiczendem, bis zu acht Direktoren (nach Dienstalter) und drei von der Gesamtheit der Gerichrsmitglieder gewählten Richtern. Seit dem Gesetz zur Änderung der Präsidialverfassung vom 26. s. 1972 ist für alle Gerichte in allen Gerichtszweigen ein Präsidium vorgesehen. Es bestcht aus dem Präsidenten als Vorsitzendem und je nach Gerichtsgröße acht oder vier planmäßigen Richtern, die gewählt werden (je zur Hälfte vorsiczende und beisitzende Richrer). Das heißr, daß die mehrheitlich nicht beförderzen Richter be- 
nachteiligı sind. Bei kleinen Gerichıcn bilden die Richıcr gemeinsam das Präsidium. Das Reformgeserz hat ferner Anhörungsrechte der Richter vor der Vornahme oder Änderung der Geschäftsvertcilung festgeschrieben: für alle Richeer vor Enescheidungen über ihre Zuteilung oder Zuständigkeit, für Vorsitzende außerdem vor der jährlichen allgemeinen Geschäftsverteilung und vor Änderungen, die ihren Spruchkörper bexühren. Die Forderung reformfreudiger Richter nach Öffenclichkeit der Präsidiumssitzungen für Richter, wiewohl sicherlich mit dem Gesetz vereinbar, hat sich nicht durchgescizt. Die Bildung der Spruchkörper innerhalb der Gerichte war und blieb Sache der Justizverwaltung (z. B. in Baden-Württenberg aufgrund $\$$ s des Ausführungsgesctzes zum Gerichtsverfassungsgcsetz vom 16. 12. 1975).

Dic Emennung der Richter geschieht im Bund durch den zuständigen Miniscer zusammen mit einem Richterwahlausschuß. In den Ländern erfolgt sie teils durch unterschiedlich zusammengesezze Richrerwahlausschusse aufgrund von Geseczen aus der Zcit zwischen 1962 und 1971, teils durch den zuständigen Minister. Die Richier sind daran durch Präsidialräte beteiligı, welche die Länder nach dem Deurschen Richtcrgesetz vom 8. 9. 196r für jeden oder für mehrere Gerichiszweige gemeinsam einrichten müssen. Dieses Bundesrecht aus der Adenauer-Zeir schreibt nur vor, daß mindestens dic Hälite der Mitglieder (außer dem Vorsitzenden) gewählt sein müssen. Das baden-württembergische Landesrichtergesetz vom 25. 2, 1964 sah vor, daß die Hälfte der Mitglieder der Präsidialräte von den Richtern, dic andere Hälfte von Wahlmännern aus den Gerichtspräsidien zu wählen war. Nach der Neufassung dicses Landesgesetzes vom 19. 7. 1972 werden nun alle Mitglieder der Präsidialrätc von den Richtern direkt gewahlt, ebenso in einigen anderen Bundesländern. Sie müssen in Baden-Württemberg bereits fünf Jahre lang Richter auf Lebenszeit sein, eine Beschränkung, die ebenso in anderen Bundesländern gilt. Sie bedeutct, daß ungefähr für das erste Dritrel ihres Bcrufslebens die Richrer niche wählbar sind, also die jüngere Richtergeneration nic an der Personalpolicik betciligt ist, wenn man die Proberichterzeit und die vierjährige Amtsperiode der Präsidialräte berücksichtigt. Eine weitere Sicherung der aithergebrachten Standesvertretungen vor dem Eindringen neuer Sirümungen der Richterschaft in die Präsidialrätc scellı das Mehrheitswahlrecht dar, welches z. B. in Baden-Würtemberg 1972 (zur Zeir des Aufkommens der ÖTV-Gruppen von Richtern und Staatsanwältren) statr des seit 1964 bis dahin getrenden Verhältniswahlrechts eingeführt wurde. Bei der Wahl des Präsidialrates der ordentlichen Gerichtsbarkeit im Jahre 1980 beispiels veise erhielt die ÖTV-Gruppierung deshaib trocz fast $30 \%$ Scimmanceil keinen Sicz im Präsidialrat. Ähnlich sicht es in andern Bundesländern aus. In keinem Zweig des öffentlichen Dienstes gibr es für eine Personalvertrecung dieses Mehrheitswahlrecht. Eigentümlich für Baden-Würrtemberg ist dic Ausbildung des Richterwahlausschusses als eines bloßen Konfliktausschusses für den Fall, daß Minister und Präsidialrat sich nicht einigen.

2. Ein wesentliches Element der Unterordnung der Richterschaft unter die Exckutive ist die Regelung der Besoldang. Deshalb war das Streben nach mehr Unablängigkeit hicr auch besonders stark. Die Richter verlangten, daß ihre Bczahlung in geringerem Maße an Beförderungen gekoppelt ist. Dieser Wunsch wurde 1970/71 durch Geserze in den Bundesländern zum Teil erfüllr. Das hessische Geserz über die Amtsbczüge der Richter und Staatsanwälte vom 4. 3. 1970 ließ nur noch drei Besoldungsstufen für Richter zu. Die anderen Bundesländer machten die Richter vom Beförderungswesen fínanziell insofern unabhängiger, als sie $\times$ Durchstufungen * bei der Besoldung einführten: der Richter im Eingangsamt gelangtc automatisch mir steigendem Dienstalter von A i bis nach A is, also bis zur Besoldung eines mehrfach beförderten höheren Beamten. Der Richter im ersten Beförderungsamt wurde ebenso automatisch von A is weiter bis nach A 16 durchgestuft. Seit der Grundge- 
setzänderung vom 18. 3. 1971 hat der Bund die konkurrierende Gcsetzgebungskompetenz für die Besoldung, die er durch das 2. Gesetz zur Vereinheitlichung und Neuregelung der Bcsoldung von 23. 5. 1975 ausübte, indem $\mathrm{cr}$ eine besondere, von der Regelung für Beamte unterschiedene Besoldung * $R$ * für Richter und Staatsanwälte schuf, $n \mathrm{R}$ I « entspricht etwa dem „durchgestuften $\alpha$ Richter im Eingangsamt, ^R 2 \& dem sdurchgestuften * einmal beförderten Richter (Vorsitzende von Spruchkörpern, Beisitzer an Obergerichten). Dic Besoldungssteigerungen richten sich nach dem Lebensalter, nicht nach einem individucllen Dienstalter. $R_{3}$ bis $R$ rogilt für die Richter und Staatsanwältc in höheren Funktionen. Es besteht also weiterhin cin Zusammenhang zwischen Besoldung und Beförderung. $E_{r}$ ist abcr für die meisten Richter ( $R$, und $R 2$ ), dic ohnehin keine höheren Funktionen crrcichen, abgeschwächt. Denn der Abstand der Besoldung zwischen $R$ I und $R 2$ ist gering. Wegen des Gcldes muß deshalb die innere Unabhängigkeit des Richters nicht berührt sein.

3. Dasselbe Gesetz vom 26. 5. 1972, welches die Gerichtspräsidien reformiertc, ordnete auch die Dienstbezeichnungen der Richter neu. An die Stelle der althergebrachten beamtengleichen "Räten-Titcl trat die Bezeichnung "Richter», die Direktorcn und Senatspräsidenten wurden zu "Vorsitzenden Richrern . Hinzu trat die Bezcichnung des Gerichts, an dem der Richter cine Planstelle har. Die Präsidenten der Gerichıc behieiten diesen Tircl; nur rückte das Gerichı hinter den Präsidenten-Titcl. Aber das Bundesverfassungsgericht verlangte durch seinc Entscheidung vom 27. 6. 1974 eine Korrektur des Gesetzes; auf ihr beruht die Wiedereinführung der Dienstbezeichnungen "Vizepräsident « und "Direktor « für bestinmte Stelleninhaber durch Gesetz vom 22. 12. 1975 .

4. Das Bestreben nach ciner Reform der Juristenausbildung fand seinen Niederschlag im Memorandum des Loccumer Arbeitskreises vom 8. Oktober 1969' und in den Leitsätzen der Arbeitsgemeinschaft Sozialdemokratischer Juristen zur Ausbildungsreform ${ }^{6}$. Durch Gesetz vom 10. 9. 1971 wurdc in das Deutsche Richtergesetz $\$$ sb eingefügr, eine Experimentierklausel $\propto$, welche den Bundesländern auf zehn Jahre die Einrichtung einer cinphasigen Ausbildung erlaubte. Die Aufspairung in Scudium und Referendariat, in Theorie und Praxis, konnte nun versuchsweisc gemildert oder aufgegeben, das Prüfungswesen verändert werden. Die prakcischen Erfahrungen der Länder soliten dann zu einer bundeseinheitlichen Neurcgelung hinführen. Von der Experimentierklausel machten sieben Bundesländer Gebrauch, beginnend zwischen 1971 und r 975 . Das Münchner Modell (mchr oder weniger übernommen von BadenWürttemberg, Nordrhcin-Wcstfalen und Rhcinland-Pfalz) zielt auf Effizienz ab, während das Hamburger Modell (ähnlich prakuziert auch in Niedcrsachsen) auf Änderung der Ausbildungsinhalte Werc legt. Dic Bremer Universität geht mit der Einbezichung der Sozialwissenschaften in dic Juristenausbildung am weitesten. Zur gleichen Zeit wurden Dauer und Modalitäten der hergebrachten zweiphasigen Ausbildung ebenfalls gcändert ( $\$$ sa und sd des Deutschen Richtergesetzes). Die Experimentierklausel wurde durch Gesecz vom 16.8.1980 verlängerr: A usbildungsgänge nach eincm der neucn Modelle können nun bis zum is. 9. 1984 aufgenommen werden.

Wenn sich in den Ictzten Jahren etwas in der Justiz geändert hat, dann am wenigsten durch die Veränderungen der Juristcnausbildung. Pluralistischer ist die Jusciz deshalb geworden, weil sie Zeitströmungen und Veränderungen des allgemeinen Bewußtseins nicht ganz von sich abwehren konntc. Von inhalelichen Ausbildungsre-

5 Jurisuische Schulung 1969, S. 999 .

6 Reche und Politik 1970, 5. $41 ; 1972$, S. 28 ff. 
form-Modellen halicn sich inzwischen nur noch inselhafte Reste. Durch Geserzentwurf vom 27. 8. 1982 versuchte die sozialliberale Bundesregierung, aus den Modellgängen wenigstens einen mehrfachen Wechsel zwischen Studium und Praxis (nämlich während der Grund- und der Schwerpunktausbildung) in eire bundeseinheirliche Regelung hinüberzuretten. Es sieht aber so aus, als würden wir mit dem zeitichen Ablauf der Expcrimencicrklausel einfach wieder in die alte Juristenausbildung zurückfallen, vielleicht mit kleinen Änderungen. So hat es der oReformausschußa der Justizministerkonferenz beschlossen, so steht es im Gesetzentwurf der Länder Niedcrsachsen und Schleswig-Holstein, und so wünscht es der Bundesarbeitskreis der CDU-Juristen.

ऽ. Zu den Reformen, die das Publikum, die Gerichtsbenutzer, berühren, gehört die Abscbaffung der kleinen Amesgerichte. Sie geschah in allen Flächenstaaten der Bundesrcpublik in den frühen siebziger Jahren. In Baden-Würtemberg wurden beispielsrueise durch Gesetz vom 10. I. 1974 elf von 1 19 Amrsgerichte aufgehoben - ein Schritt auf dem von Bundesjustizminister Jahn anvisicrten Weg, dic Amts- und Landgerichrc einander anzunähern und zu vereinigen. Die unterschicdliche Gerichtsbarkeit für das Massenpublikum (Amtsgcrichte; Landgerichte als zweite Instanz) und für die etwas besseren Leute, den Mittelstand, (vom Landgerichr bis zum Bundesgerichtshof) wäre auf diese Weise zu einem einheitlichen drcistufigen Gerichrszug verschmolzen rvorden. Die Verlagerung der Familiensachen von den Landgerichten zu den Amtsgerichten ab 1. 7. 1977 war ein weitercr Schritt in dieser Richtung, aber ansonsten blieb die "vierstufige ordentiche Gerichtsbarkeic, die eine Arr Klasseneinteilung darstellre, erhatren.

6. Der allgemeine Gerichtssiand, an dem cin Käufer, Darlehensnehmer, sonstiger Schuldner verklagt werden kann, ist sein Wohnsitz oder Aufenthaitsort. Weir verbreicet waren Vereinbarungen, welche dem Lieferanten, der Bank oder dem sonstigen Gläubiger erlaubien, am Ort seines Sitzes zu prozessieren. Von diesem Sítz weit enternr wohnende Schuldner, besonders aus Bevölkerungskreisen, die ohnehin schon das Gericht nicht gern betreten, ließcn unabhängig von der Rechtslage massenweise Versäumnisurteile gegen sich ergehen. Nach der Gerichtsstandsnovelle vom 21.3. 1974 is eine vom allgemeinen Gerichtsstand abweichende Zustänóigkeitsvereinbarung nur noch ausnahmstveise zulässig, z. B. unter Kauflcuren oder nach Entscehen der Rechtsstreitigkeit oder für das Mahnverfahren. Die zuletır genannte Ausnalime isc eine Schwäche des Geseczes, da es Schuldner gibt, wclche cine Korrespondenz mit einem auswärtigen Gericht nichı aufnehmen.

7. Das Gesecz zur Enilastung der Landgerichte und Vereinfachung des Protokolls vom 20. 12. 1974 hob die Wertgrenze für die Zuständigkeir der Amtsgerichte in Zivilsachen von is 500 auf $3000 \mathrm{DM}$ und dic Mindestsumme für die Einlegung einer Bcrufung von 200 auf j00 DM. Außerdem übertrug es dem Einzelrichcer am Landgericht (also einem einzelnen Mirglied der Zivilkammer) die Entschejdungsberugnis anstelle der Kammer in erstinstanzlichen Sachen, wenn diese sic ilım überantwortete, während der Einzelrichcer bis dahin nur einen Kammercermin vorzubereiten harte. Der vorbercirende Einzelricheer wurde in Bcrufungssachen (also bei den Berufungskammern des Landgerichts und beim Oberlandesgericht) neu fakultaciv eingeführt. Das Gesecz diente dazu, der in der damaligen Wirtschaftsflaute ansceigenden Prozeßflut Herr zu werden. Es senkte den personellen Aufwand pro Verfahren und vermehrce die Kompetenzen der bis dahin bloß beisitzenden (nicht zum Vorsitzenden Richter befördercen) Kammermitglieder. Es war ein Schritr in der Richtung, alle erscinsianzlichen Sachen durch den Einzelrichrer behandeln zu lassen, bis hin zur Umwandlung des vierstufigen Aufbaus der ordentlichen Gerichtsbarkeir zu einem dreisrufigen. 
8. Das umfängliche Kostenrecbts-Änderungsgesetz vom 20.8.1975 war reformerisch nur in einem sehr beschränkten Sinne: es richrete die Kostenregeln zu für den Einsatz der elektronischen Datenverarbcicung in den Gerichten und für dic Anwendung des Kostenrechts durch Beamte des mittleren state des gehobenen Dienstes. Ein übersichticher Gebührenkatalog trat an die Stelle vieler of komplizierter Einzelvorschriften; für Massengeschäfte (z. B. bei der Vollstreckung) wurden Festgebühren eingeführt; andere Gebühren wurden gescrichen. Das Problem der Kosten als Zugangsbarrierc vor der gerichelichen Rechesdurchsetzung schob der Gesetzgeber hingegen weiter vor sich her. Schlimmer: er verschärfte es, indem er die Gerichtsund Anwaltsgebühren für nicdrige und besonders stark für mitulere Streitwerte orhöhte.

9. Das Gesetz zur Beschleunigung und Vereinfachung gerichtlicher Verfahren vom 3. 12. 1976 fügtc in dic Zivilprozeßordnung hauptsächlich die Rechtsgrundlagen für das schon mehrere Jahrc experimeniell praktizierte Prozessieren mic einem einzigen Verhandlungstcrmin ("Stuttgarter Modell ") ein sowic Änderungen des Mahnverfahrens und der vorläufigen Vollstreckbarkcit. Das Gericht soll nach der neuen ZPO in der Regel jede Sache nach einem "Hauptrermin entscheiden und diesen so vorbereiten, daß der Partcivortrag komplet und die Beweismitrel prassenc sind. Damit die Parteien dazu beitragen, sind die Verspätungsrcgeln verschärf́t worden. Man kann sic ungefähr so zu einer Faustregel zusammenfassen: wer ein Angriffs-oder Verceidigungsmittel später vorbringr, als es ihm möglich gewcsen wäre, wird damit nur gehört, wenn es das Verfabren nicht verzögert.

Diese Verfahrensbeschleunigung ist schr oft eine Wohltar für denjenigen, der scin Recht durchsetzen muß. Sic gerär aber häufig in Konflikt mit dem Hauprzweck des Prozesses: die wrichrige "Lösung zu finden. Das Gesetz ist ein eindeutigas Signal für eine hohe Bewertung schneller Verlahrensabwicklung. Selbst wenn die Prozeßparteien cinvernehmlich den Prozcß zeitlich strecken wollen, z. B. Terminsvcrlegungen wünschen, soll das Gericht sich darüber hinrvegsetzen. Der Antrieb zur Beschleunigung resultierı offenkundig nicht aus dem Wunsch, Interessen der Prozcßparteien zu erfüllen. Es ist dic zunehmende Bclastung der Gerichte mit Prozessen bẹi angespannter Personalsituarion, die die Justizpolitiker in der ökonomischen Dauerkrise nach Beschleunigungsgesetzen greifon lassen.

Beschleunigung und Auffinden der richtigen Konfliktlösung sind im übrigen nicht die einzigen Leitsterne beim Prozessieren. Es kann z. B. für dic Parteien gur oder auch schlecht scin, wenn das Geriche schon zum ersten Verhandlungstcrmin das volle Zeugenaufgebor, den oder die Sachverständigen, alle Bcweismittel herbeizitiert. Der Streit wird gelegentlich dadurch verticft, der Eingriff in die materiellen und ideellen Interessen Unbcteiligter unnötig ausgeweiter, der Kostenaufwand erhöht. Viele Rechtssachen erledigen sich icichter und besser mit halber Mobilisierung der Justizmittel. Der „frühe erste Hauptermin « mit Präsenz aller Beweispersonen is deshalb nicht für alle Prozesse gecignet.

Das Mahnverfahren ist durch die "Vereinfachungsnovelle aú die maschinelle Bearbeitung durch EDV-Anlagen an zentralen Amtsgerichten vorbercitet worden. Es ist stark formalisiert, die mit menschlichem Denken verbundene Schlüssigkeitsprüfung ist abgeschaffr, das Gesetz trcibu die Mahngerichte ausdrucklich zu eiliger Bearbeitung an. Mahnverfahren können jetzt also in prakeisch unbegrenzten Mengen ablaufen. Verzögerungen durch Schlendrian oder durch den Druck starken Arbeitsanfalls werden keinem Schuldner mehr Aufschub bringen. Er bekommt außerdem als Rechtsbelchrung nur den Hinweis auf die Möglichkeit des Widerspruchs innerhalb von zwei Wochen, $\$ 692$ I 3 ZPO (obwohl auch ein spärerer Widerspruch den noch nicht verfügten Vollstreckungsbescheid noch abwenden kann, $\$ 694$ I 
ZPO), nicht aber die Belehrung über den Einspruch gegen Vollstreckungsbescheide.

10. Erst am 1. 1. 198 2 tracen schließlich zwei Stücke Justizreform in Kraft, die den eigentlichen Kern der lang diskutierten Reformen bilden sollien: Das Gesecz über die Prozeßkostentrilfe vom 13.6. 1980 und das Gesetz über Rechesberatung und Vertetung fiur Bürger mit geringem Einkommen vom 18.6. 1980. Das Prozeßkostenhilfegeserz löste das bisherige "Armenrecht " für alle Gerichtszwcige ab. Es gewährt je nach Einkommen Kosten ŕreiheit und unentgeltliche Anwaltsbeiordnung oder das (völlige oder teilweise) Aufbringen der Kosten in Razen. Eine Tabelle berücksichrigt die Untcrhalespflichien bei der Berechnung der Berechigung zur Prozeßkostcnhilfe. Danach prozessiert z. B. ein Lediger ohne Kinder bei einem Nerroeinkommen bis 850 DM frei; bci einem Einkommen von $2400 \mathrm{DM}$ muß er momatlich Raten von $520 \mathrm{DM}$ aufbringen, ein alleinverdienender Ehegatte mit zwei Kindern 80 DM. Geblieben ist die Beschränkung der Prozeßkostcnhilfe auf Fallc, die vom Gericht als aussichtsreich eingestuft werden, und die Verpflichiung, beim Mißerfolg die Anwaltskosten des Gegners zu tragen.

Das Beratungshilfegesetz gewälurt außerhalb von Gerichrsverfahren stark verbilligien Anwalusrat (nämlich zu $20 \mathrm{DM}$, die aber auch erlassen rverden können) aú Staatskosten, wenn man zu dem Personenkreis gehört, der nach der Tabelle für die Prozeßkostenhilfe von eigenen Beiträgen zum Prozessieren befreit isr. Der oben genanntc Familienvater zum Beispiel bekomme diese Hilfe bei einem Nettoeinkommen bis 1850 DM. In Bremen und Hamburg bleibr es bei den traditionellen öffentlichen Rechrsbcratungsstellen. Ausgenommen von der Beratungshilic sind das Arbeirs- und das Sozialrecht, die Domänen gewerkschaftlichen Rechtsschutzes.

Die Einführung dieser bciden Geserze für Minderbemitrclte gab die Bahn frei für eine weirere Erhöhung der Anwaltsgebühren durch das Gesetz vom 18.8. 1980. Erhard Blankenburg ${ }^{7}$ har beschrieben, daß sich das Bcracungshilfegesecz sehr viel langsamer in die Praxis umsetzt, als man erwartet hatte. Eine "sozialc Anwalischafte, die sich auf Bedürfrige spezialisiert, kann sich wohl nur langsam bilden.

xx. Die Strafjustiz ist durch den Wegfall der stark welcanschaulich fundierten Tatbestände besonders im Sexualstrafrecht moderner geworden. Viel an enrwüdigender und für kein wirkliches Interesse in der Gesellschaft erforderlicher Menschenverfolgung ist damit abgcschafft worden. Dic Liberalisierung des Demonstrationsstrafrechts hat damals einen ganzen Schwung betroffener vorwiegend junger Menschen zum aktiven Engagement in den Institutionen des Staates und der Gesellschaft veranlaßr. Die geserzlichen Anpassungen im Wirtschafts- und Umweitstrafrecht sind sicherlich als Anfang und Signal geeignet, wenn sic zu einer entsprechenden Praxis der Sraatsanwaltschaften und Gerichte führen. Der Eindruck verdüstert sich aber, wenn wir an die Auswirkungen des Terrorismus auf Gesetzgebung, Rechtsprechung und Strafvollzug denken. Manches an stratlicher Reaktion war notwendig und niche anders zu erwarten. Insgesamt waren aber sicherlich nichı nur dic 1976 geschafienen und 1981 wieder aufgehobenen $\$ \$ 882$ und 130a StGB zu weit gehende Eingriffe in das ohnehin of schon die Freiheit der Meinungsäußerung und der policischen Becätigung berührende Strafrecht. Der Strafprozeß trägt dic Male der Terrorismus-Abwehr - man denke an Verreidigcrausschluß und -überwachung, an das Verbor der Verteidigung mehrerer Täter, an das Kontakisperregesctz.

Der Strafvollzug schließ̂lich ist die Probe auis ganze System staaclichen Strafens. Wesentiche Reformlorderungen sind unerfüllt. Und dic Zurückdrängung der kur- 
zen Freiheitsstrafen wird überrollt von den vielen geldlosen Verurteilten, die ibre Ersazzfreiheitsstrafen antreten. Auch die Strafrechtsreformen erhalten sich also nichr aus sich selbst heraus, sondem kamen und kommen zwischen die Mühlsteine des Staatsschuczes und der ökonomischen Krise.

Diese Übersicht läßt die Familienrcchtsreform sowic Besonderhciten in der Entwicklung der Arbeits- und Verwaltungsgerichtsbarkeit aus. Deshalb ist auch die folgende Gesamtbetrachtung über die Justizreform ergänzungsbedürftig.

\section{Die Justiz am Ende der Reformphase}

I. Einen Teil der Interessen der Richter hat die Justizreform erfüllt: die Mirwirkungsrechte und die Besoldung sind heute günsciger geregelt ais früher. Bei der Geschäfrsverceilung in den Gerichten ist die Mitwirkung der Richter stark ausgeprägt. In der Personalpolitik der Länder üben die Präsidialräce der Richter durch Informiertheit und Mitsprache Einfluß aus. Die den Richterrären verblecibenden Sachgebiete der Personalvertretung liegen besonders in den Ländern, wo es Stufenvertretungen gibc (Bezirks- und Haupcrichterräte), dem Zugriff der Richterschaf́ an sich offen, soweit sic Neigung zur Ausübung ihrer Rechre spürt. Sowcit in den Mitwirkungsorganen aktivc Richter sitzen, arbeiten sie dort nicht ohne Erlolg. Oft führen die Richtcrräte allerdings ein Schatendasein. Mit den Präsidialträten tcilen sie die Schwäche, dic in der Nichtöffentlichkeit ihrer Arbeit und der Verschwiegenheitspflicht ihrer Mirglieder liegr. Impulse gehen von diesen Gremien deshalb in der Regel nicht aus. Die Mehrzahl der Richter vermißs das aber auch niche.

Zu den crfüllten Richterwünschen gehört auch dic maßvolle Abkoppelung der Besoldung vom Beförderungswesen. Die erste Beförderung wirkt sich auf die Besoldung nur schrvach aus. Und wenige Richter haben ohnebin die Aussicht, das zweite Beförderungsant zu erreichen. Wer sich dem Karrierewesen nicht unterwerícn will, ist also finanziell dadurch gegenüber der Mehrzahl der Kollegen nur wenig benachteiligr.

Zusammenfassend kann man sagen: Soweit dic Richterinteressen stark formulier und vorgebracht worden sind, har sie die Jusizreform einigermaßcn berücksichtigt. Die Forderung nach Abbau des hierarchischen Systems blieb allerdings im wesentlichen unerfüllt, da der Reformdruck auch von seiten der Richecr zu schwach war. In der Justizverwaltung, im Beförderungswesen, im Verhälenis der Gerichesinstanzen zueinander und in der Arbcir der Spruchkörper finden wir dieselben Strukturen rvie vor is Jahren vor. Bis zu einem gewissen Grade war und ist das nichic zwangsläufig, denn mit ihren Mitwrirkungsechten könnten die Richter hie und da verändernd eingreifen. Eben dies unterbleibc aber meistens. Je emanzipativer ein Reforminteresse unter Richtern war, desto weniger wurde es verwirklicht. Je individueller dic zu befriedigenden Wünsche waren, desto stärker drückten die Richter sie durch und desto größer waren ihre Erfolgc.

1. Welche Verbesserungen brachten Reformen für die Klientel der Justiz, für das Volk? Das Problem des «Zugangs" zur Ziviljustiz ist sicherlich im Laufe der Rcformjahre für die Mituelschichten und die aktiveren Teile der Unterschichten gemildert worden. Von der neuen Gerichtsstandsregelung bis zur Beratungs- und Prozcßkostcnhilfe haben Reformmaßnahmen Erleichterungen der Rechtsaussübung gebrachr. In dieser Richrung wirken auch die Verbesserungen des Verbraucherrechrs. Zwiespältig sind aber diejenigen Veränderungen der Ziviljustiz zu bewerten, die das Durchsetzen von Forderungen auf cine Wcise crleichtern, dic cendenzicil die Unter- 
schichten benachteiligt, wie etwa die Computerisierung des Mahnverf́ahrens aufgrund der ZPO-Novelle vom 9. 12. 1976 (Erleichterungen für Massen- und Großgläubiger) oder dic durch dasselbe Gescrz cingeführte Prozeßbeschleunigung, die nur für aufgeklärte Beklagte gefahrlos ist. Daß der Zivilprozeß stromlinienförmiger wurde, gehı auf Kosten der unteren Schichten, Ihnen hätte eher ein Gerichtswesen dicnen können, wie cs Rasehorn vorgeschwebr hat: cin dichtes Nerz kleiner Beraiungsstellen mit informeller Arbeitsweise. Aber die Organisationsreform verlief in entgegengeseizter Richiung.

Erhard Blankenburg ${ }^{8}$ hat dic Einschränkungen beschricben, mir denen das Recht überhaupt Hilfe bietet: Abhängige verzichten oft auf die Rechrsausübungen wegen der sozialen Nebenfolgen von Prozessen, sozial Schwache haben oft gar keine Rechtsposition für ihre Lebensbedürfnisse, und weitcre Grenzen des prakcischen Rechtsschutzes. Den Randschichten, die von diesen Einschränkungen betroffen sind, hatce und hat auch ein verbesserter Zivilprozeß niches zu bieten. Wenn das von vornherein klar war, so könnte man für die vorausschbar allcin begünstigte Mittclschicht und obere Unterschicht die Reformen der Ziviljustiz und des Verbraucherrechts als geglückt ansehen.

Dic ökonomische Strukturkrise hat aber eine empfindliche Schwäche der Reformen enthüllt: sie waren ausgerichter auf cinen Wachstumskapicalismus und verlieren mit dcssen Wanken an Werr. Jetzt erfaßt dic ökonomische Krisc auch dic Gerichte selbst. Bei zunehmender Belastung der Gerichte ohne Pcrsonalvcrmehrung zeigen sich Beschleunigung und Vereinfachung des Verfahrens von ihrer gefährlichen Seite. Denn nkurzer Prozeßa geht in aller Regel zulasten des Schwächeren. Selbst wer matcrielle Rechte hat, wer cigentlich "Recht hat", kann es bei ungeschickter Ausnutzung der verknappten Justizressourcen leichter verlieren als in Zeiren ruhigen und geduldigen Prozessicrens. Die Erleichterung für die von den Reformen Begünstigten helien nicht dagegen, daß sich die ökonomischen Tendenzen zulasten der Sch wachen auswirken.

Insgesamt zeigt sich deutlich die Bcgrenztheit der Reformziele, die angestevert wurden. Wir müssen sehen, daß die Reformen uns nicht auf die Probleme der achıziger Jahre vorbercitec haben. Dazu hätten sic ciefer greifen muissen. Die Kritik der heurigen Probleme der Gesellschaft enchält die Feststcllung, daß dic eigentlich notwendigen Reformen ausgeblicben sind, auch in der Justiz. Der Justizapparar und seine Arbeitsweise hätten sich nur wesendich ändern können durch mächtige Anstöße aus der Gesellschaft heraus, welche sich in einer Gesamtreform befunden hätcc. Durch alle Poren hindurch hätte der Wandel in die Justiz cindringen müssen, dann hätte sie niclı die gleiche bleiben können. Solche Nörigung zum Wandcl blieb aber aus. Sie lag vor allem auch nicht in den Anläuien zur Ausbildungsreform. Hans Albrecht Hesse ${ }^{9}$ hat die begrenzten Erwarrungen geschildert, die man von vornherein in Ausbildungsreformen als Motor zur Jusrizreform nur setzen durfire. Der Druck der Praxis isı stärker als ein Gestaltungswille von Berufsanfängern. Allein beharrliches Bcmühen ausrcichend vieler Bcrufspraktiker könntc etwas ändern, soweic das soziale und staaliche Umfeld der Justiz das zulassen. Wir sehen, wie die Reformansäzze in der Juristenausbildung rorgedrückt wurden und werden.

Einc günstiger verlaufende Reformepoche hätte durch Änderungen der Juscizstrukturen die Justiz so umgestalten sollen, daß vor dem Hintergrund der deurschen

\footnotetext{
$8 \times$ Rechlshilfebedürfnis und Rechtsberalung* in: Jahrbuch für Rechtssoziologıe und Rechlstheone. Band $V, 1978, S, 231$

9 × Routine und Reforma in: Zur Theoric der Reform, hersusgegeben von M. Greiffenhagen, Karlsruhe 1978. S. $57,23 \mathrm{ff}$.
} 
Geschichte dic NS-Anfälligkeir der dritcen Gewalt hättc bervä)tigt werden können, dadurch daß die Justiz nach außen autonomer und nach innen weniger hierarchisch geworden wäre. Wie die siebziger Jahre verliefen, ist die Justiz zwar kein schlechteres, doch auch kein besseres Bollwerk gegen undemokratische und unfreiheitliche Tendenzen geworden. Ein einheitliches "Urteil "über die Justiz läßr sich sicherlich niche sprechen. Aber vielfach öffnct sie sich den Zumurungen, welche wirtschaftliche und politische Mächte an sie herantragen. Die dritte Gewalt ist den anderen Gewalten auf wichtigen Sektoren dienstbar auch dort, wo sie Anlaß zur Verweigerung von Legalitär und Legitimitäı hätre.

Wic gehr es weiter? Zunächst mit dem gemeinsamen Versuch, Rechte und Reformstücke zu erhalten. Aber das Bild gesellschaftlichen Wandels, welches in den sechziger und siebziger Jahren an den Wänden hing, ist verblaßt. Wir werden nie mehr an dic damaligen Reformstrategien anknüpfen können. Es gibt keinen rechten Sinn, die Strukturmängel der Jusciz mit denselben Rezepten in der Hand kurieren zu wollen, wie man sich das am Anfang der Reformepoche dachce. Denn dazu war is Jahre lang Zeit und geschichtliche Gelegcnheit. Unsere Nöte der nächsten Jahre sind anders, als sie sich fortschrittlichen Gesellschaftspolitikern darbocen, die nach der Ära Adcnaver in die Zukunft (die jerzt Vergangenheit isc) aufbrachen. Die Suche nach ganz anderen, neuen Bildern gescllschafclichen Wandels läuft. Die Juristen haben da keine Lcitstellung, sie brauchen viclinchr ihrerseits Gespür dafür, welchen Strömungen sie sich öffnen müssen. In der Gesellschaft werden Elemente alternaliver Modelle sichtbar. Schon die Sterilitä́ technokratischer Macherei deckte nicht mehr alles zu, und die wende - Philosopheme bringen es jetzr auch nicht fertig.

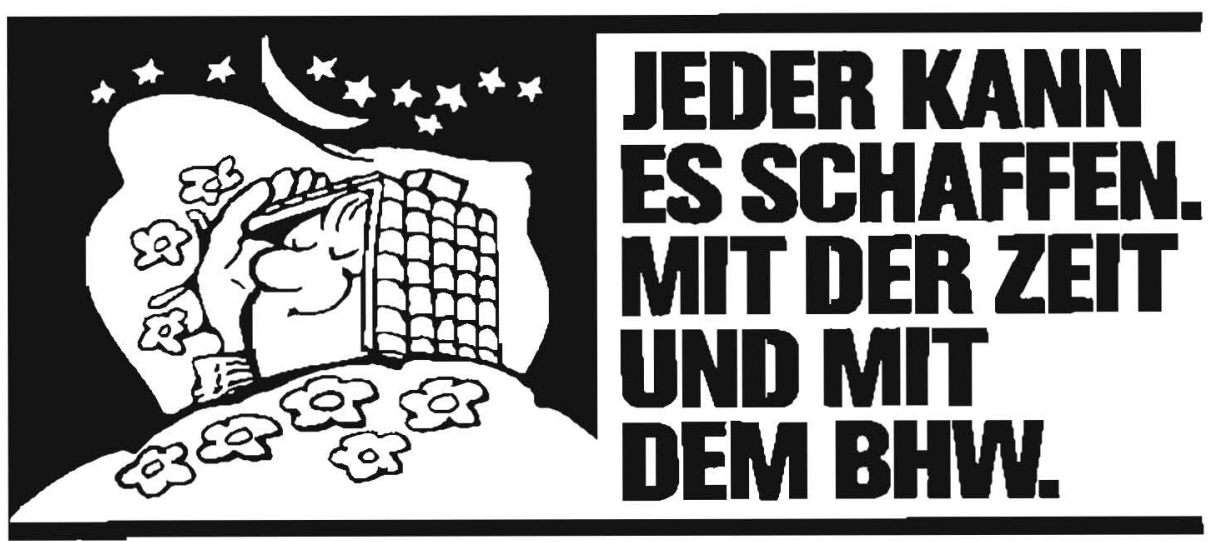

Das eigene Hcion - wer davon eräume, der sollec jetzr etwas dafür run: Bausparcon.

Bausparen ist fast dic cinzıge Möglichketr, an güinstiges Baugelld zu konmon.

Bausparen isc dic an höchsten vom Stalat getörócrte Sparform.

Batusparen beim BHW ist jerzt noch attraktiver: zum Beispicl durch $4 \%$ Guthabenzinsen im. BHW-Vernögunsbiklungs-Farif.

Sprechen Sie deshalb gleich mic Ihrem BHW-Berater, oder rufen Sie ihn an. Das BHW stche in jedem örlichen Telefonbuch.

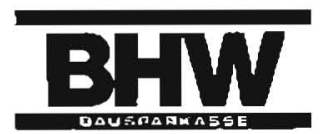

Auf uns baut der öffentliche Dienst. 